\title{
LncRNA PCAT-1 upregulates RAP1A through modulating miR-324-5p and promotes survival in lung cancer
}

\author{
Na Huang, Wenjing Dai, Yunhui Li, Jian Sun, Chunlan Ma, Wancheng Li
}

Pulmonary and Critical Care Medicine, The First Affiliated Hospital of Chengdu Medicine University, Chengdu, Sichuan, China

Submitted: 11 December 2018

Accepted: 26 February 2019

Arch Med Sci 2020; 16 (5): 1196-1206

DOI: https://doi.org/10.5114/aoms.2019.84235

Copyright $\odot 2019$ Termedia \& Banach

\begin{abstract}
Introduction: Lung cancer is the malignant tumor with the fastest increase in morbidity and mortality and the greatest threat to human health and life. Long non-coding RNA (IncRNA) is emerging as an important regulator in many cancers. Recently, it was found that IncRNA prostate cancer associated transcript 1 (PCAT-1) was up-regulated in lung cancer, playing oncogenic roles. However, the underlying regulatory mechanism of PCAT-1 remains unknown. Material and methods: The expression levels of PCAT-1 and miR-324-5p were analyzed by real-time PCR, and RAP1A expression was determined by western blotting. RNA pull-down, luciferase and western blotting assays were used to examine the target relationship between PCAT- 1 and miR-324$5 p$ or that between miR-324-5p and RAP1A. The functional effects of PCAT-1 and miR-324-5p were examined using cell viability and cell apoptosis assays. Results: PCAT-1 overexpression remarkably promoted cell proliferation and suppressed cell apoptosis. Mechanistic investigations demonstrated that PCAT-1 can interact with miR-324-5p and repress its expression, thereby increasing the expression of its target RAP1A. Additionally, rescue experiments revealed that PCAT-1 served as an oncogene partly through sponging miR-324-5p and upregulating RAP1A in lung cancer cells.

Conclusions: Our findings demonstrate that on account of the dual function of pro-proliferation and anti-apoptosis, PCAT-1/miR-324-5p/RAP1A may be novel candidates for application in the diagnosis, prognosis and therapy of lung cancer.
\end{abstract}

Key words: lung cancer, IncRNA PCAT-1, miR-324-5p, RAP1A, cell survival.

\section{Introduction}

As one of the most common respiratory malignancies, lung cancer remains a leading cause of cancer-associated deaths [1]. Although the underlying molecular mechanism of lung cancer remains largely unknown, it is generally considered that a combination of multiple abnormal gene expression, such as oncogene activation and tumor suppressor gene inactivation, may lead to the occurrence and development of lung cancer [2]. However, existing findings are not sufficient to fully explain the pathogenesis of lung cancer. With advances in molecular biology and gene diagnosis, more and more evidence indicates that long noncoding RNAs (IncRNAs) are important regulators for the expression of a large number of genes and play an extensive role in human diseases, including the pathological processes of tumorigenesis [3].

\author{
Corresponding author: \\ Wancheng Li \\ Pulmonary and Critical \\ Care Medicine \\ The First Affiliated \\ Hospital of Chengdu \\ Medicine University \\ 278 Middle Section \\ of Baoguang Avenue \\ Xindu District \\ Chengdu, Sichuan \\ Province, China \\ Phone: +8602883016637 \\ Fax: +8602883016637 \\ E-mail: \\ WanchengLi1298@163.com
}


Dysregulated IncRNA prostate cancer associated transcript 1 (PCAT-1) has been found to be involved in the pathological processes of various cancers. Initially identified from the RNA high throughput sequencing of prostate tissue and cells, PCAT-1 is upregulated in prostate cancer and promotes the proliferation of prostate cancer cells by regulating cMyc expression [4]. High expression of PCAT-1 is related to distal metastasis and poor prognosis among colorectal cancer patients [5]. PCAT-1 can regulate the expression of Cyclin-dependent kinase inhibitor $1 \mathrm{~A}$ (CDKN1A), a potent cyclin-dependent kinase inhibitor, and promote proliferation and metastasis of gastric cancer cells [6]. By regulating the Wnt/ $\beta$-catenin pathway, PCAT-1 promotes the progression of cholangiocarcinoma [7]. Additionally, the expression of PCAT-1 was overexpressed in liver cancer, and patients with low expression of PCAT- 1 have better survival prognosis [8]. Based on the above studies, PCAT-1 was found to be up-regulated in a variety of cancers, including gastric cancer, liver cancer and colorectal cancer, which played an oncogenic role. Recently, Zhao et al. [9] indicated that PCAT-1 was overexpressed in non-small cell lung cancer and could promote cell proliferation and metastasis. However, the underlying mechanism of PCAT-1 in lung cancer still remains unclear.

Increasing evidence indicates that as small noncoding RNAs with a length of 18-22 nt, miRNAs play a vital function in cancer development [10]. Previous studies reported that miR-181b inhibited B-cell lymphoma-2 (Bcl-2) expression and increased the susceptibility of small cell lung cancer $\mathrm{H} 446$ cells to cisplatin [11]. Existing findings suggested that some cancer associated genes were regulated by miR-324-5p. Chen et al. reported that miR-324-5p protects against colitis-associated tumorigenesis by modulating the expression of CUE domain-containing protein-2 (CUEDC2), a ubiquitin-binding motif-containing protein, which could modulate ER- $\alpha$ protein stability through the ubiquitin-proteasome pathway [12]. Gu et al. found that miR-324-5p up-regulation inhibited the proliferation and invasion of colorectal cancer cells by inhibiting the expression of ELAV-like protein 1 (ELAVL1), whose function is to stabilize mRNAs in order to regulate gene expression [13]. Additionally, other verified targets of miR-324-5p included CUEDC2 in breast cancer, specificity protein 1 (SP1) in cervical cancer, Skp1Cullin1-F-box $\beta$-transducin repeat-containing protein $\left(\mathrm{SCF}^{\beta-\mathrm{TCP}}\right) \mathrm{E} 3$ ligase in multiple myeloma and Tetraspanin 8 (TSPAN8) in gastric cancer [14-17]. In lung cancer, Lin et al. revealed that miR-324-5p was significantly overexpressed [18]. However, the precise role of miR-324-5p in lung cancer development is still largely unknown.
The small GTPase superfamily plays a crucial role in many biological processes which including cell proliferation [19]. As a member of the Ras tumor gene family, RAP1A (growth factor receptor-bound protein 2) was recently demonstrated to play a role in ERK/MAP kinase signaling and integrin activation and promote the development of several cancers [20, 21]. Activation of RAP1A increased cell migration and invasion and enhanced the rate and incidence of metastasis in a xenograft mouse model of prostate cancer [22]. The findings of Sayyah et al. implicated a Rap1A/ $\beta 1$ integrin pathway, activated downstream of G-protein-coupled receptor stimulation and RhoA in glioblastoma cell proliferation [23]. In addition, it was reported that the altered microtubule dynamics resulting from reduced RAP1A expression in lung cancer has an effect on abrogating normal microtubule function, leading to cell death [24]. Whether the dysregulated expression of RAP1A in lung cancer is related to miRNA regulation needs further investigation.

In the present study, through miRDB analysis, it was predicted that there are seed binding sites between PCAT-1 and the miR-324-5p mature sequence. Additionally, as predicted by TargetScan software, miR-324-5p may target RAP1A 3'-UTR. Therefore, we speculate that PCAT-1 may play a role in lung cancer by regulating the axis of miR-324-5p/RAP1A. The function and regulatory mechanism of PCAT-1, miR324-5p and RAP1A in the cell proliferation process of lung cancer were systematically studied, which may contribute to the improvement of diagnosis, prevention and treatment of lung cancer.

\section{Material and methods}

\section{Cell culture}

A549 cells were cultured in the F12 medium supplemented with 10\% FBS (Thermo Fisher Scientific, Rockford, IL, USA), and were incubated in a humidified atmosphere of $5 \% \mathrm{CO}_{2}$ at $37^{\circ} \mathrm{C}$. The cells were purchased from the Institute of Cell Biology at the Chinese Academy of Sciences (Shanghai, P. R. China).

\section{Plasmid constructs}

The sequence of IncRNA PCAT-1 was amplified using PCR with a human genomic DNA from A549 cells as the template. One Step Cloning Kit ClonExpress II (Vazyme Biotech, Nanjing, P. R. China) was used. The corresponding reverse primer for the IncRNA PCAT-1 was 5'-CGGGTCGACTCTAGAGGTACCTAGGCTCAAACACACATTTATTCATC-3', and the forward primer was 5'-CGAGAATTCACGCGTGGTACCACACATGGATATTGGATATCTGCATA-3'. The sequence of IncRNA PCAT-1 was then inserted into the Kpnl site of the $\mathrm{PCl}$ mammalian expression 
plasmids (Thermo Fisher Scientific) and verified using sequencing. Two effective sequences of shRNAs (Sangon Biotech) for PCAT-1 were proven and inserted into a shRNA eukaryotic expressing vector. One sequence is 5'-TTGAGTACAAAGAGCTACCTATGGTTCAAGAGAGGTATCCATCGAGAAACATGAGTTTTTTTTT-3', and the other sequence is $5^{\prime}$ CTTCCCATGTGCCTCTAAGTGTTCAAGAGAGTGAATCTCCGTGTACCCTTCTTTTTTT-3'. Additionally, the p-MIR-reporter plasmid (Promega, Madison, WI, USA) containing the $3^{\prime}$-UTR of RAP1A was constructed according to the above methods. The corresponding reverse primer for RAP1A 3'-UTR was 5'-TAGGTTTAAACAGTTAAGCTTACTGTTTAAAATGGGAAGGTTTATTAAG-3', and the forward primer was 5'-AAAAGATCCTTTATTAAGCTTGTGAATCTCCGTGTACCCTTCG-3'. The binding-site mutant luciferase plasmid (binding site: GGAUGC replaced by CCTACG) was also transfected as a control.

\section{Cell transfection}

A549 cells were transfected with PCAT-1 expressing plasmid (PCAT-1), PCAT-1 shRNA expressing plasmid (PCAT-1 sh1, PCAT-1 sh1), miR-324-5pmimics or their corresponding controls (Control, NC sh or NC mimic) using Lipofectamine 2000 transfection reagent (Thermo Fisher Scientific). Different kinds of cells were collected for qRT-PCR and luciferase reporter analysis $24 \mathrm{~h}$ after transfection, cell apoptosis and western blotting assays $48 \mathrm{~h}$ after transfection or cell viability assay 0,24 , 48, 72, $96 \mathrm{~h}$ after transfection.

\section{Cell viability assay}

A549 cells were respectively transfected with PCAT-1 expressing plasmid, control plasmid or PCAT-1 expressing plasmid and miR-324-5p. The cells were then seeded onto 96 -well plates at a density of $6 \times 10^{3}$ cells per well. After $0,24,48$, 72 , and $96 \mathrm{~h}$, the number of viable cells was detected using WST-8 staining with Cell Counting Kit-8 (CCK-8, Dojindo, Tokyo, Japan).

\section{Cell apoptosis assays}

A549 cells were transfected with PCAT-1 expressing plasmid (PCAT-1), PCAT-1 shRNA expressing plasmid (PCAT-1 sh1, PCAT-1 sh2), PCAT-1 expressing plasmid and miR-324-5p mimics (PCAT-1 + miR-324-5p) or their corresponding controls (Control or NC sh). Forty-eight hours after transfection, the cells were washed with PBS, fixed with $70 \%$ ethanol, incubated with RNase A (Takara, Shiga, Japan) for $30 \mathrm{~min}$ at $37^{\circ} \mathrm{C}$, and incubated with propidium iodide (PI) and Annexin $V$ (Sigma-Aldrich) for $10 \mathrm{~min}$. After that, the cells were subjected to a DNA content test using a FACSCalibur system (BD Biosciences, San Jose,
CA) and the ModFit_LT software was used to analyze the results.

\section{Protein extraction and western blotting}

Proteins from A549 cells were extracted using RIPA lysis buffer (Sigma-Aldrich). The total protein content was quantified using a BCA protein assay kit (Thermo Fisher Scientific). The antibodies for the detection of apoptosis associated proteins consisted of Bcl-2, Cleaved caspase-3, Cleaved PARP, internal reference GAPDH and corresponding secondary antibodies (Cell Signaling Technology, 1 : 1000). The membranes were incubated with horseradish peroxidase-conjugated anti-mouse IgG or goat anti-rabbit (Thermo Fisher Scientific, 1 : 2000) after incubation with the primary antibodies overnight at $4^{\circ} \mathrm{C}$. The GAPDH level was used as an internal control for protein expression.

\section{RNA extraction and quantitative real-time PCR (qRT-PCR) assays}

The TRIzol reagent (Thermo Fisher Scientific) was used to extract total RNA from the cultured A549 cells. A SYBR PrimeScript miRNA RT-PCR Kit (Takara, Shiga, Japan) was used to examine miRNA levels; the qRT-PCR assays were carried out according to the manufacturer's protocols. The expression of miR-324-5p was normalized to the expression of the U6 snRNA level. The reverse primer for miR-324$5 p$ was 5'-CTCAACTGGTGTCGTGGAGTCGGCAATTCAGTTGAGCACCAATG-3', and the corresponding forward primer was 5'-ACACTCCAGCTGGGCGCATCCCCTAGGG-3'. The sequences of 5'-AACGCTTCACGAATTTGCGT-3' and 5'-CTCGCTTCGGCAGCACA-3' were used to amplify the U6 SnRNA.

\section{RNA pull-down assay}

The RNA pull-down assay was performed as previously described [25]. Briefly, biotin-labeled IncRNA PCAT-1 containing the miR-324-5p binding site was transcribed from PCAT-1 expressing plasmid with the Biotin RNA Labeling Mix (Roche, Indianapolis, IN, USA) and T7 RNA polymerase (Promega), treated with RNase-free DNase I (Promega) and purified with an RNeasy Mini Kit (Qiagen, Valencia, CA). Biotinylated RNA was denatured at $95^{\circ} \mathrm{C}$, put in ice, left at room temperature to allow secondary structure formation, and then incubated with whole-cell lysates from A549 cells at $25^{\circ} \mathrm{C}$. The IncRNA PCAT-1-RNA complexes were isolated with Streptavidin agarose beads (Thermo Fisher Scientific) and the pull-down miRNA-324-5 $p$ was detected by qRT-PCR.

\section{Luciferase reporter assay}

When the cell confluence was at 70-80\% in each well of 6-well plates, A549 cells were 
co-transfected with the firefly luciferase reporter plasmid (1 $\mu \mathrm{g}), \beta$-galactosidase expression vector (0.5 $\mu \mathrm{g}$, Promega) and miR-219-5p-mimic/ NC-mimic $(100 \mathrm{pmol})$ or PCAT-1/Control $(0.5 \mu \mathrm{g})$ using Lipofectamine 2000. The protein was extracted $24 \mathrm{~h}$ after transfection, and luciferase activities were tested using a Luciferase Reporter Assay System (Promega) according to the manufacturer's instructions.

\section{Statistical analysis}

Experiments were conducted in triplicate and repeated at least three independent times. Results in this study are expressed as the mean \pm standard error of the mean (SEM). Differences between two groups were analyzed using Student's $t$-test. The comparison between multiple groups was performed using one-way ANOVA. $P<0.05$ was considered significant. ${ }^{*} P<0.05 ;{ }^{* *} p<0.01$; ${ }^{* * *} p<0.001$.

\section{Results}

Overexpression of IncRNA PCAT-1 promotes cell proliferation and inhibits apoptosis of A549 cells

As the up-regulation of IncRNA PCAT-1 is a frequent event in lung cancer, we first examined PCAT-1 expression in four lung cancer cell lines (A549, SPC-A-1, NCl-H1734 and H460) and a human bronchial epithelial cell line (16HBE) using qRT-PCR. The levels of PCAT-1 in four lung cancer cell lines were higher than that in the 16HBE cells. Among the four lung cancer cell lines, A549 expressed a relatively high level of PCAT-1 (data not shown), which is largely consistent with the reported results [9]. Thus, A549 was chosen for the investigation of PCAT-1 in lung cancer. QRT-PCR assays were performed to test IncRNA PCAT-1 level after transfection with PCAT-1 expressing plasmid (PCAT-1), PCAT-1 shRNA expressing plasmid (PCAT-1 sh1, PCAT-1 sh2) or their corresponding controls (Control or sh NC). The results revealed that the IncRNA PCTA-1 level was markedly increased after transfection of PCAT-1 compared to the Control cells, and was significantly decreased after transfection of the PCAT-1 sh1/PCAT-1 sh2 compared to the NC sh cells (Figure $1 \mathrm{~A}$ ). The cell viability and apoptosis assays were next examined. As Figure 1 B shows, overexpression of PCAT-1 significantly promoted cell proliferation, and the cell growth was inhibited after the level of PCAT-1 was knocked down. The results of flow cytometry showed that the upregulation of PCAT-1 decreased the percentage of apoptotic cells, whereas downregulation of PCAT-1 increased the proportion of apoptotic cells (Figure $1 \mathrm{C}$ ). Additionally, after PCAT-1 overexpression, anti-apoptotic protein $\mathrm{BCl}-2$ expression was markedly upregulated, and the levels of cell apoptosis associated proteins (Cleaved caspase-3, Cleaved PARP) were significantly decreased. The results presented the opposite trend after downregulation of PCAT-1 (Figure $1 \mathrm{D}$ ). These results indicated that InCRNA PCAT-1 can promote cell proliferation and inhibit apoptosis in A549 cells.

\section{IncRNA PCAT-1 interacts with miR-324-5p and downregulates its expression}

In lung cancer cells, the up-regulation of InCRNA PCAT-1 promoted cell proliferation and inhibited cell apoptosis. However, the molecular mechanism for this remains indistinct. Therefore, the potential miRNA targeted by PCAT-1 was predicted by bioinformatics analyses. Through the bioinformatics prediction method (miRDB, http:// www.mirdb.org), we found that PCAT-1 could probably interact with miR-324-5p. We next focused attention on the interaction between miR-324-5p and PCAT-1 in the cell growth and apoptosis of A549 cells. As Figure 2 A shows, there was perfect base pairing between the binding seeds of miR-324-5p and PCAT-1, which is marked in blue. Additionally, biotinylated PCAT-1 harboring the miR-324-5p binding site was synthesized in vitro and the biotin-avidin pull-down assay was performed. The qRT-PCR results demonstrated that miR-324-5p was significantly enriched in the RNA immunoprecipitation (RIP) pulled down by biotinylated PCAT-1 (Figure 2 B). Next, we determined the expression level of miR-324-5p using qRT-PCR after over- or down-expressing PCAT-1. As Figure $3 \mathrm{C}$ shows, overexpression of PCAT- 1 caused significant downregulation of miR-324-5p, and the miR-324-5p level was increased after PCAT-1 was knocked down. These findings together revealed that PCAT-1 can inhibit the level of miR-324-5p by targeting its binding seeds.

\section{IncRNA PCAT-1 upregulates the protein level of RAP1A through inhibiting miR-324-5p expression}

In A549 cells, PCAT-1 could influence the expression level of miR-324-5p. Nevertheless, the role of miR-324-5p in A549 cells remains largely unknown. We next predicted target genes of miR324-5p using bioinformatics analyses. By means of TargetScan, we found that RAP1A is probably a potential target of miR-324-5p. Next, we studied the regulatory relationship between RAP1A and PCAT-1/miR-324-5p in lung cancer cells. Accordingly, the seed binding sites between RAP1A and miR-324-5p were depicted in Figure $3 \mathrm{~A}$; they are marked in blue. qRT-PCR results showed that the miR-324-5p level increased about 140-fold after transfection with miR-324-5p mimics (Fig- 


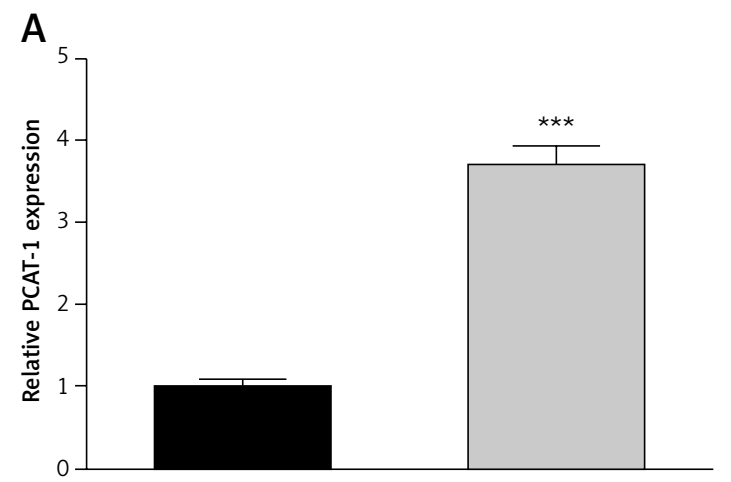

Control

\section{B}

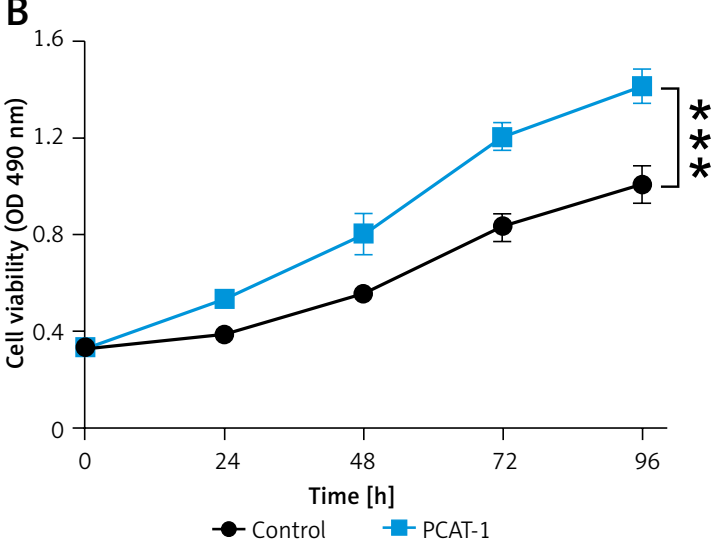

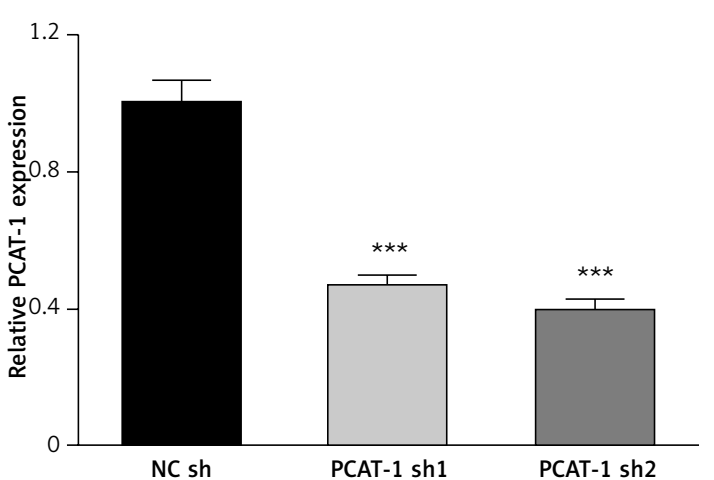

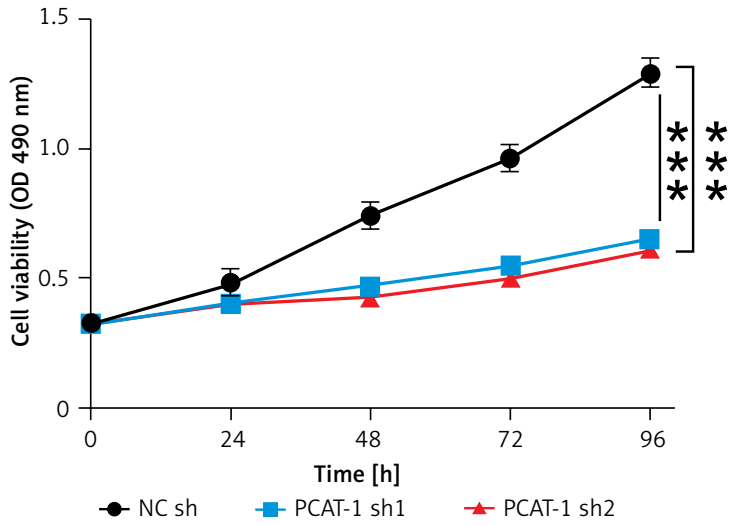

NC sh $\quad$ PCAT-1 sh1
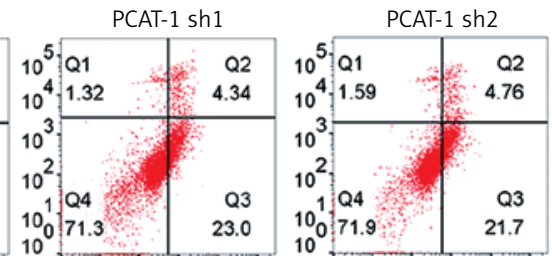

100 21.7

$\bar{a}$
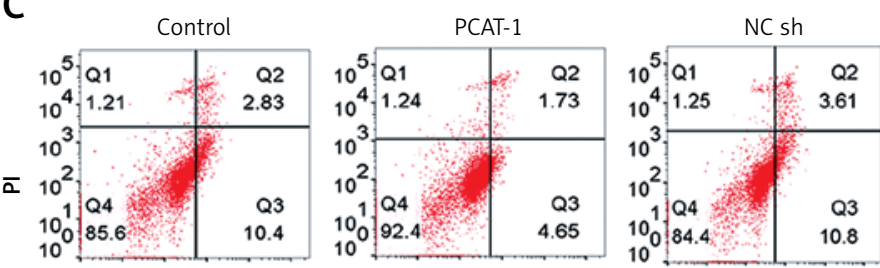

$10^{0} 10^{1} 10^{2} 10^{3} 10^{4} 10^{5} \quad 10^{0} 10^{1} 10^{2} 10^{3} 10^{4} 10^{5} \quad 10^{0} 10^{1} 10^{2} 10^{3} 10^{4} 10^{5} \quad 10^{0} 10^{1} 10^{2} 10^{3} 10^{4} 10^{5} \quad 10^{0} 10^{1} 10^{2} 10^{3} 10^{4} 10^{5}$

Annexin V
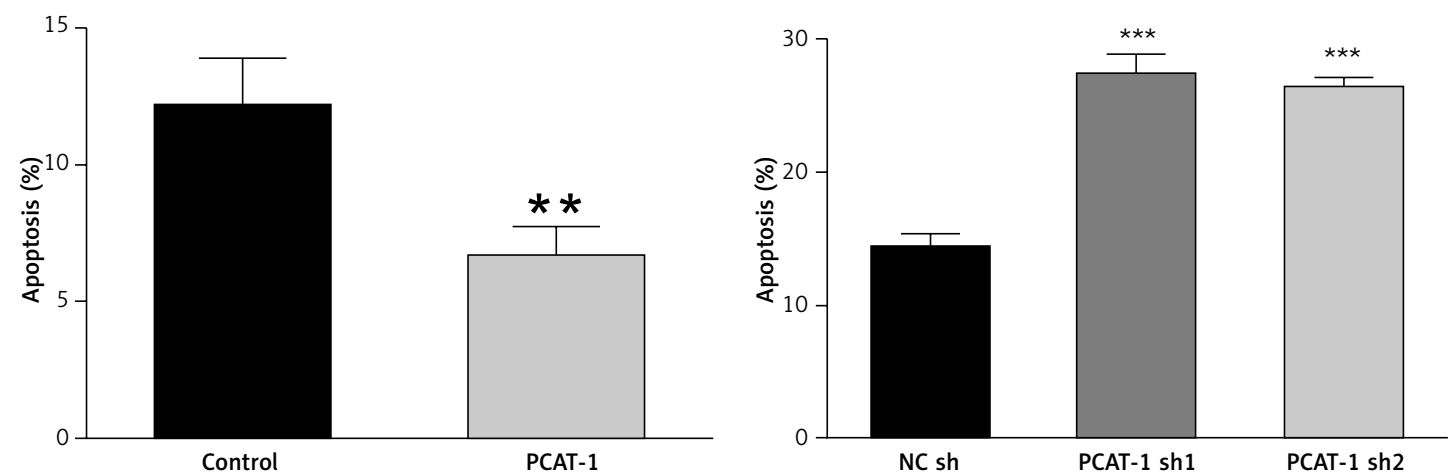

Figure 1. Overexpression of IncRNA PCAT-1 promotes cell proliferation and inhibits apoptosis of A549 cells. A - Relative IncRNA PCAT-1 expression levels after transfection with PCAT-1 expressing plasmid (PCAT-1), PCAT-1 shRNA expressing plasmid (PCAT-1 sh1, PCAT-1 sh1) or their corresponding controls (Control or NC sh), as determined using qRT-PCR. B - Growth curves of A549 cells after transfection with PCAT-1 expressing plasmid (PCAT-1), PCAT-1 shRNA expressing plasmid (PCAT-1 sh1, PCAT-1 sh1) or their corresponding controls (Control or NC sh). Measurements of the cell growth rate were obtained using a CCK-8 kit. The experiments were performed three times. C - Cell apoptosis analysis of A549 cells after transfection with PCAT-1 expressing plasmid (PCAT-1), PCAT-1 shRNA expressing plasmid (PCAT-1 sh1, PCAT-1 sh1) or their corresponding controls (Control or NC sh, left). The proportion of cell apoptosis was analyzed and presented as a histogram (right). The experiments were repeated three times 

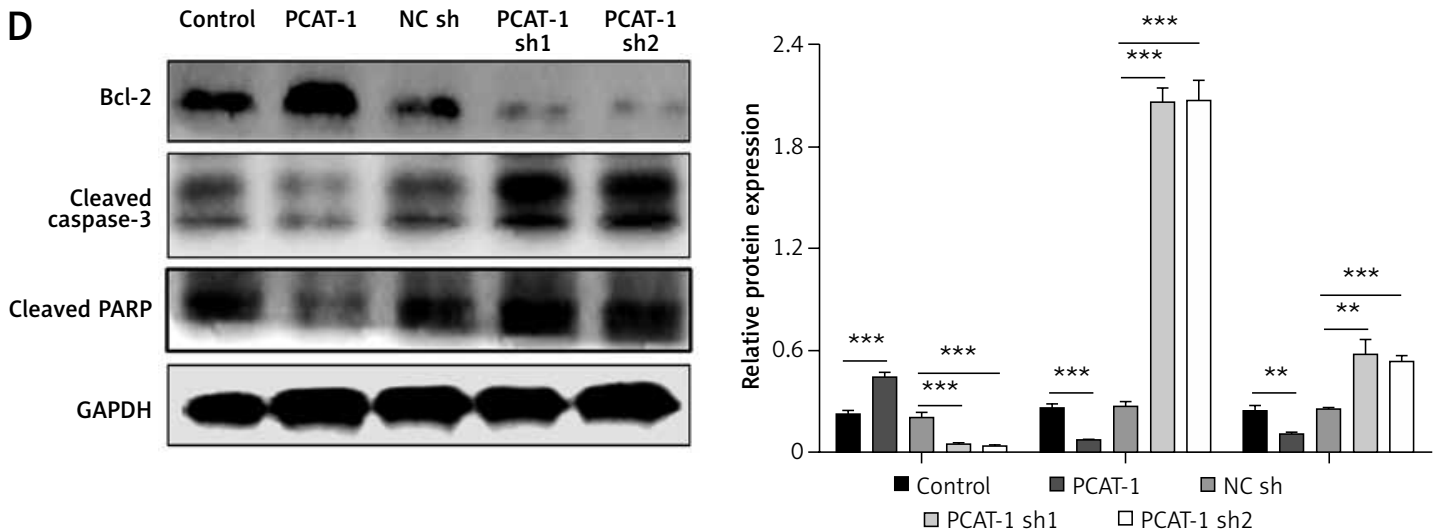

Figure 1. Cont. D - The protein level of Bcl-2, Cleaved caspase-3 and Cleaved PARP in A549 cells after transfection with PCAT-1 expressing plasmid (PCAT-1), PCAT-1 shRNA expressing plasmid (PCAT-1 sh1, PCAT-1 sh1) or their corresponding controls (Control or NC sh), as determined using western blotting (left). Relative quantitative results were determined by Image $J$ and shown as a histogram (right)

The data are expressed as mean \pm SEM, ${ }^{* * *} p<0.001$.

A

hsa-miR-324-5p 3'-UGUGGUUACGGGAUCCCCUACGC-5'

LnCRNA PCAT-1
C

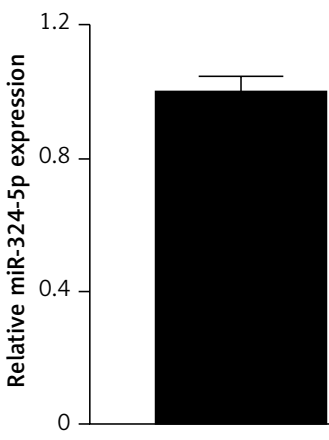

Control
5'-TCCAGCCACCTCTTGGGATGCACACGTTTC-3'
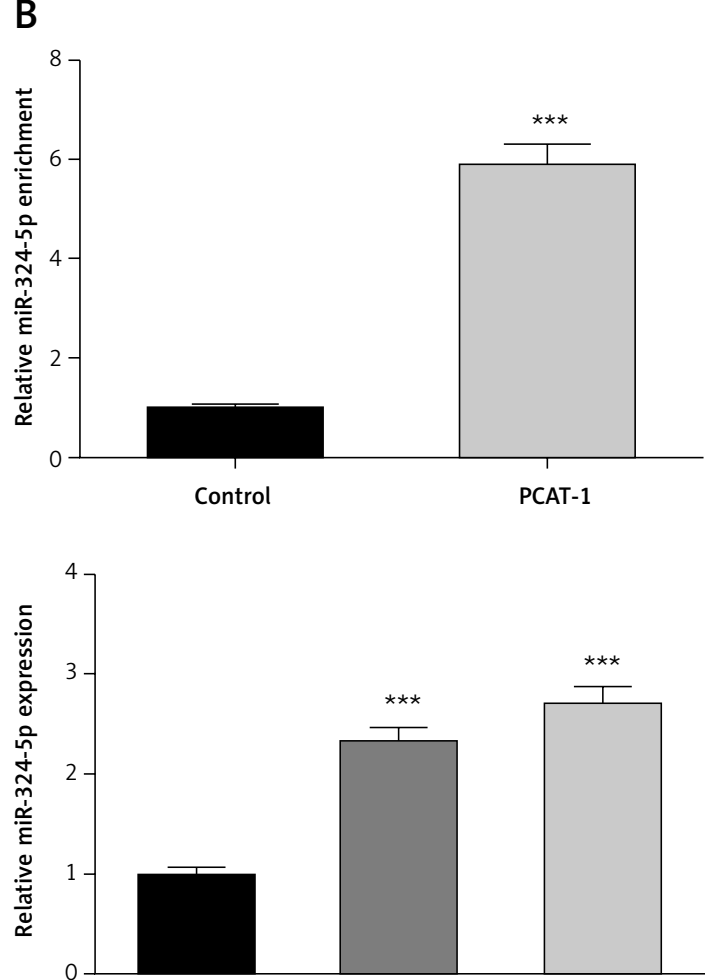

NC sh

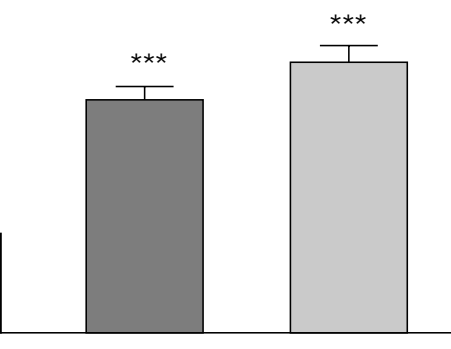

PCAT-1 sh1

PCAT-1 sh2

Figure 2. IncRNA PCAT-1 reduces the expression of miR-324-5p through binding to miR-324-5p. A - LNCipedia and miRDB prediction tools identified one seed match for PCAT- 1 in the mature sequence of miR-324-5p; the predicted seed-recognition site in the miR-324-5p sequence and the corresponding PCAT-1 sequence are marked in blue. B - miR-324-5p is associated with PCAT-1. A549 cells were harvested and mixed with biotinylated PCAT-1 to perform biotin-based pull-down. miR-324-5p enrichment was tested by qRT-PCR and normalized to the control. C - Relative levels of miR-324-5p in A549 cells after transfection with PCAT-1 expressing plasmid (PCAT-1), PCAT-1 shRNA expressing plasmid (PCAT-1 sh1, PCAT-1 sh1) or their corresponding controls (Control or NC sh), as determined using qRT-PCR

The data are expressed as mean $\pm S E M$, ${ }^{* \star *} p<0.001$. 
A

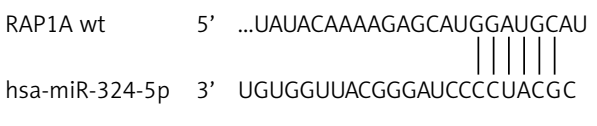

RAP1A mut $\quad 5, \quad \ldots$ UAUACAAAAGAGCAUCCUACGAU...

C

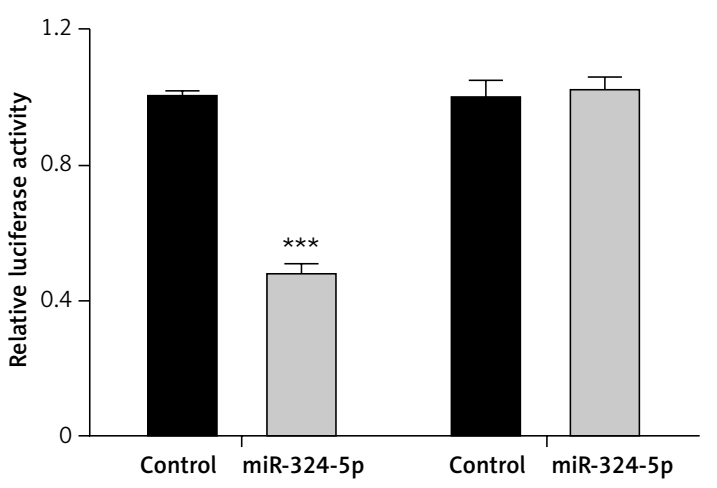

D

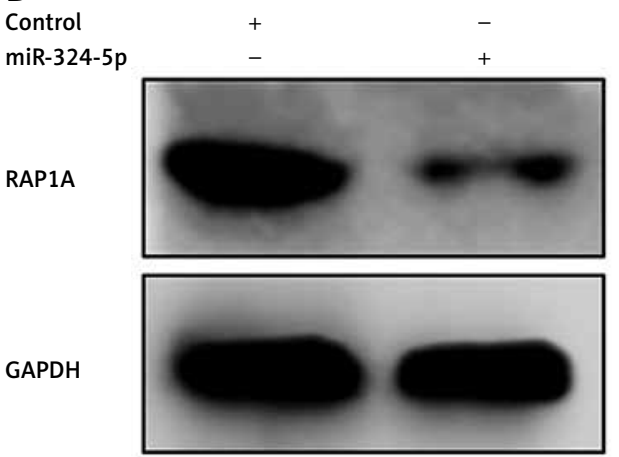

Control

PCAT-1

RAP1A

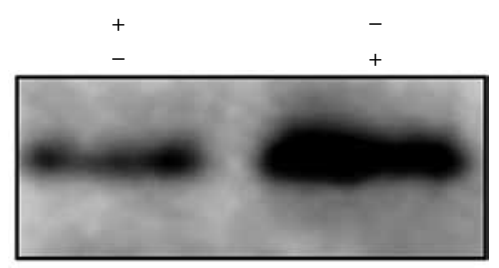

GAPDH

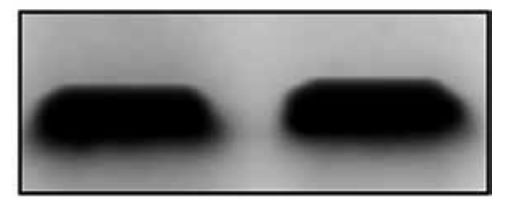

B
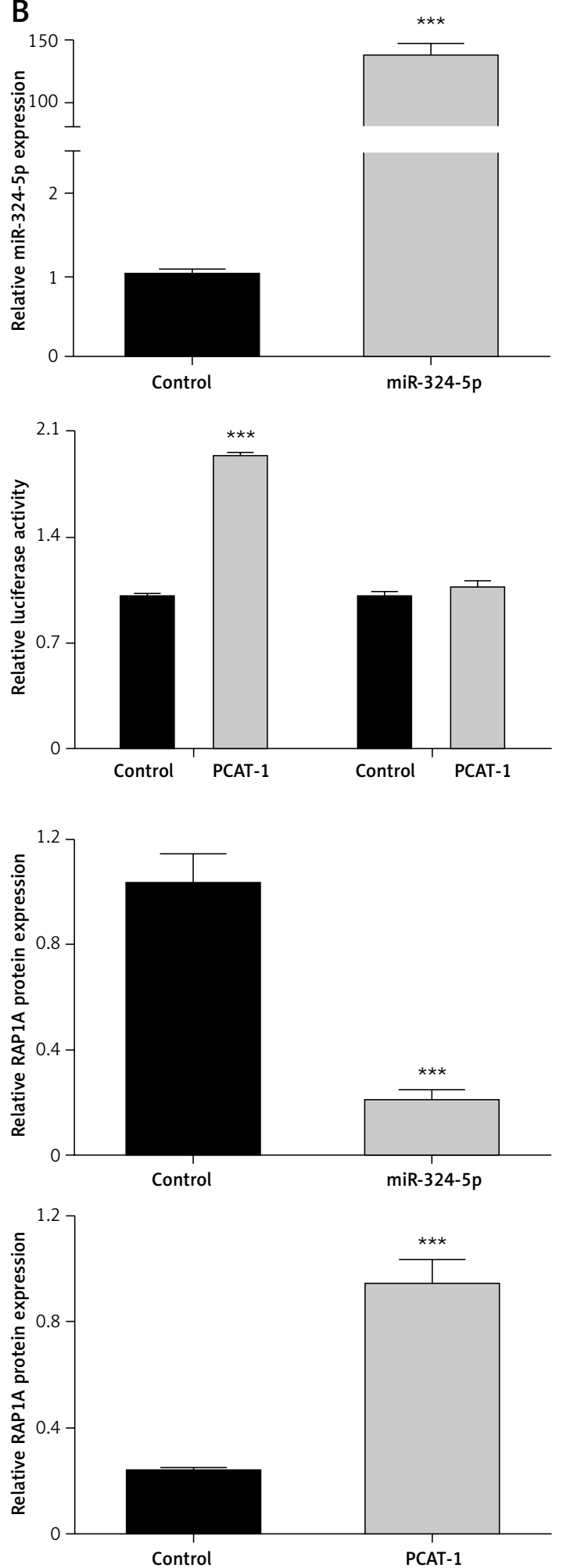

Figure 3. IncRNA PCAT-1 upregulates the protein level of RAP1A through inhibiting miR-324-5p expression. A - TargetScan prediction software identified one seed match for miR-324-5p in the $3^{\prime}$-UTR of RAP1A; the predicted seed-recognition site in the RAP1A mRNA sequence and the corresponding miR-324-5p sequence are marked in blue. B - Relative levels of miR-324-5p in A549 cells after transfection with miR-324-5p mimics or the corresponding control (Control), as determined using qRT-PCR. C - Relative luciferase activity of the WT and mutant RAP1A 3'-UTR reporter plasmids were assayed in A549 cells after transfection with miR-324-5p mimics (miR-324-5p), PCAT-1 expressing plasmid (PCAT-1) or their corresponding controls. D - Protein level of RAP1A in A549 cells after transfection with miR324-5p mimics (miR-324-5p), PCAT-1 expressing plasmid (PCAT-1) or their corresponding controls, as determined using western blotting (left). Relative quantitative results were determined by Image J and shown as a histogram (right)

The data are expressed as mean $\pm S E M,{ }^{* * *} p<0.001$. 
ure $3 \mathrm{~B}$ ). As shown in Figure $3 \mathrm{C}$, the upregulation of miR-324-5p inhibited about $53.6 \%$ of the luciferase activity of the RAP1A 3'-UTR reporter plasmid (left), while the luciferase activity was restored after the predicted miR-324-5p binding sites in RAP1A 3'-UTR reporter plasmid were mutated (right). Moreover, overexpression of PCAT-1 led to an almost doubled increase in RAP1A 3'-UTR reporter activity in contrast with the control (left), and the effect was abolished when the predicted miR-324-5p binding sites in RAP1A 3'-UTR reporter plasmid were mutated (right), which further verified the specific interaction between miR-324$5 p$ and the $3^{\prime}$-UTRs of RAP1A (Figure $3 \mathrm{C}$ ). Next, we detected the protein levels of RAP1A after the upregulation of miR-324-5p or PCAT-1 in A549 cells. Compared with the control, upregulation of miR-324-5p led to a remarkable reduction in the protein expression of RAP1A, while the overexpression of miR-324-5p resulted in a decrease in RAP1A protein expression (Figure $3 \mathrm{D}$ ). The above findings suggested that miR-324-5p can inhibit the protein level of RAP1A by targeting its $3^{\prime}$-UTR region, and furthermore PCAT-1 can upregulate the expression of RAP1A by decreasing the level of miR-324-5p.

\section{IncRNA PCAT-1 promotes proliferation and inhibits apoptosis of lung cancer cells by downregulating miR-324-5p}

Because PCAT-1 can promote cell proliferation and inhibit apoptosis, and PCAT-1 can regulate miR-324-5p expression in A549 cells, the function of PCAT-1 targeting miR-324-5p was next analyzed. The protein expression of RAP1A was detected using western blotting after transfection with PCAT-1, PCAT-1 + miR-324-5p or the corresponding controls. As Figure $4 \mathrm{~A}$ shows, the overexpression of PCAT-1 resulted in upregulation of RAP1A protein expression, while the protein level of RAP1A was weakened after co-transfection of PCAT- 1 and miR-324-5p, compared to the cells simply transfected with PCAT-1. The cell viability results demonstrated that an obvious decrease of cell proliferation was detected in cells after co-transfection of PCAT- 1 and miR-324-5p, compared with those transfected with PCAT-1. Furthermore, flow cytometry experiments were performed to evaluate the influence of miR-324-5p on cell apoptosis by inhibiting the level of PCAT-1. As shown in Figure 4 C, PCAT-1 over-expression inhibits cell apoptosis. However, the ectopic upregulation of miR-324-5p was able to weaken the effects caused by PCAT-1 over-expression. Additionally, when analyzing the influence of PCAT-1/ miR-324-5p on the expressions of cell apoptosis related proteins (Bcl-2, Cleaved caspase- 3 and Cleaved PARP), similar results were tested after co-transfection of miR-324-5p and the PCAT-1 expression plasmid. As Figure 4 D shows, PCAT- 1 overexpression upregulated the protein expression of $\mathrm{Bcl}-2$ and decreased the protein levels of cleaved caspase- 3 and cleaved PARP in A549 cells. The overexpression of miR-324-5p weakened the expression changes of $\mathrm{Bcl}-2$, cleaved caspase- 3 and cleaved PARP caused by PCAT-1 overexpression. In short, the above findings demonstrated that PCAT-1 may promote cell proliferation and inhibit cell apoptosis, which might be through regulating the expression of miR-324-5p via an inhibition mechanism.

\section{Discussion}

It was gradually found that different kinds of biomarkers such as PD-L1, secreted phosphoprotein 1 (SPP1), amyloid precursor protein (APP), circulating fucosylation genes and circulating InCRNA XLOC_009167 were related to the prognosis of lung cancer patients [26-29]. Among them, IncRNAs can regulate gene expression at chromatin, genomic, transcription and post-transcriptional levels, playing a vital function in many cellular physiological processes and holding potential to influence disease occurrence [30]. Some IncRNAs associated with lung cancer progression have been demonstrated. For example, Huang et al. found that LINC00961 overexpression could greatly inhibit lung cancer cell proliferation and decrease the cell apoptotic ratio [31]. Cheng et al. suggested that IncRNA PRNCR1 can upregulate HEY2 to accelerate lung cancer progression by competitively adjusting miR-448 [32]. Tang et al. demonstrated that IncRNA OGFRP1 overexpression can effectively function as a competing endogenous RNA for miR-124-3p to promote the expression of its target gene LYPD3 [33]. These findings revealed that IncRNA could regulate gene expression by negatively affecting the endogenous miRNA level. Previous reports have reported that PCAT- 1 is associated with the development of some kinds of cancers, including prostate cancer, colorectal cancer patients, gastric cancer cells, cholangiocarcinoma and liver cancer [4-8]. Recently, it was found that IncRNA PCAT-1 was up-regulated in lung cancer, playing oncogenic roles. Nevertheless, the underlying regulatory mechanism of PCAT-1 in lung cancer is still unknown. In this study, our results not only suggested pro-proliferative and anti-apoptotic function of PCAT-1 but also provided a regulatory relationship between PCAT-1 and miRNA.

Using the bioinformatics prediction method (miRDB), it was predicted that there exist seed binding sequences between PCAT- 1 and miR324-5p. A previous study demonstrated that as an oncogenic IncRNA, TPT1-AS1 can function as 
A

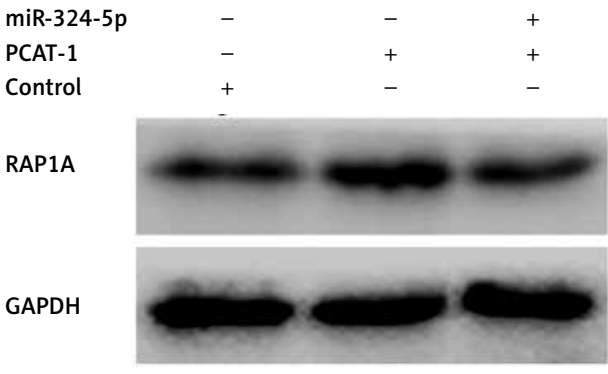

B

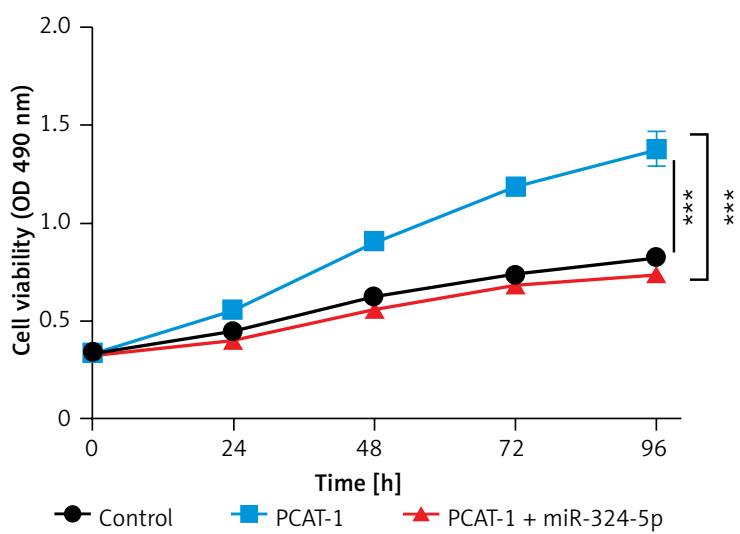

C

\begin{tabular}{|c|c|c|c|c|c|}
\hline \multicolumn{2}{|c|}{ Control } & \multicolumn{2}{|c|}{ РСAT-1 } & \multicolumn{2}{|c|}{ PCAT- $1+$ miR-324-5p } \\
\hline $\begin{array}{l}105: \longdiv { Q 1 } \\
10 ;\}: 0.67\end{array}$ & $\begin{array}{r}0.2 \\
2.35\end{array}$ & $\begin{array}{l}10 \\
10 \\
10\end{array}$ & $\begin{array}{r}02 \\
2.00\end{array}$ & $\begin{array}{l}105: \longdiv { Q 1 } \\
104: 1.06\end{array}$ & $\begin{array}{r}\text { Q2 } \\
4.13\end{array}$ \\
\hline \begin{tabular}{l|l|l|l|l|l|l|}
10 & \\
$10^{2}$ & \\
11 & $Q 4$ \\
10 & 82.1 \\
10 &
\end{tabular} & $\begin{array}{r}\text { Q3 } \\
14.9 \\
\end{array}$ & \begin{tabular}{l|l|l|}
$10^{3}$ & \\
$10^{2}$ & \\
$10^{1}$ & $Q 4$ \\
10 & 88.5 \\
\end{tabular} & $\begin{array}{r}\text { Q3 } \\
8.36 \\
\end{array}$ & $\begin{array}{l}10^{3} \\
10^{2} \\
10\end{array}$ & $\begin{array}{r}Q 3 \\
14.0\end{array}$ \\
\hline \multicolumn{2}{|c|}{$10^{0} 10^{1} 10^{2} 10^{3} 10^{4} 10^{5}$} & $10^{0} 0^{1} 10^{2}$ & $10^{4} 10^{5}$ & \multicolumn{2}{|c|}{$10^{0} 10^{1} 10^{2} 10^{3} 10^{4} 10^{5}$} \\
\hline
\end{tabular}
Annexin V

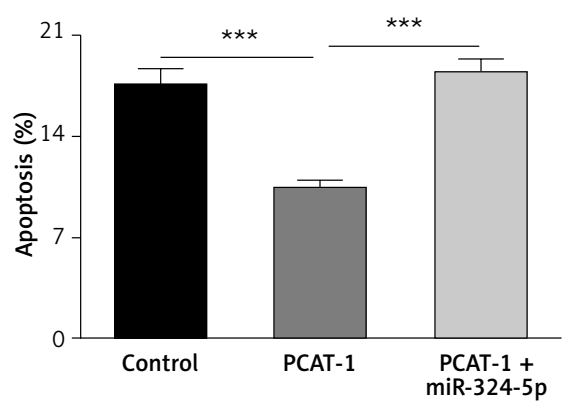

D

miR-324-5p

PCAT-1

Control

Bcl-2

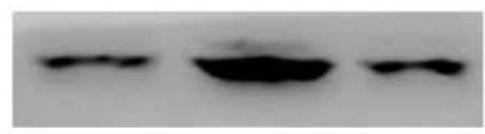

Cleaved caspase-3

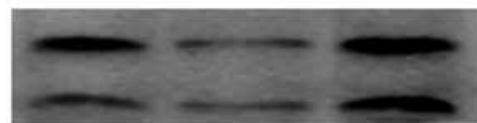

Cleaved PARP

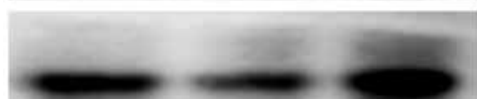

GAPDH
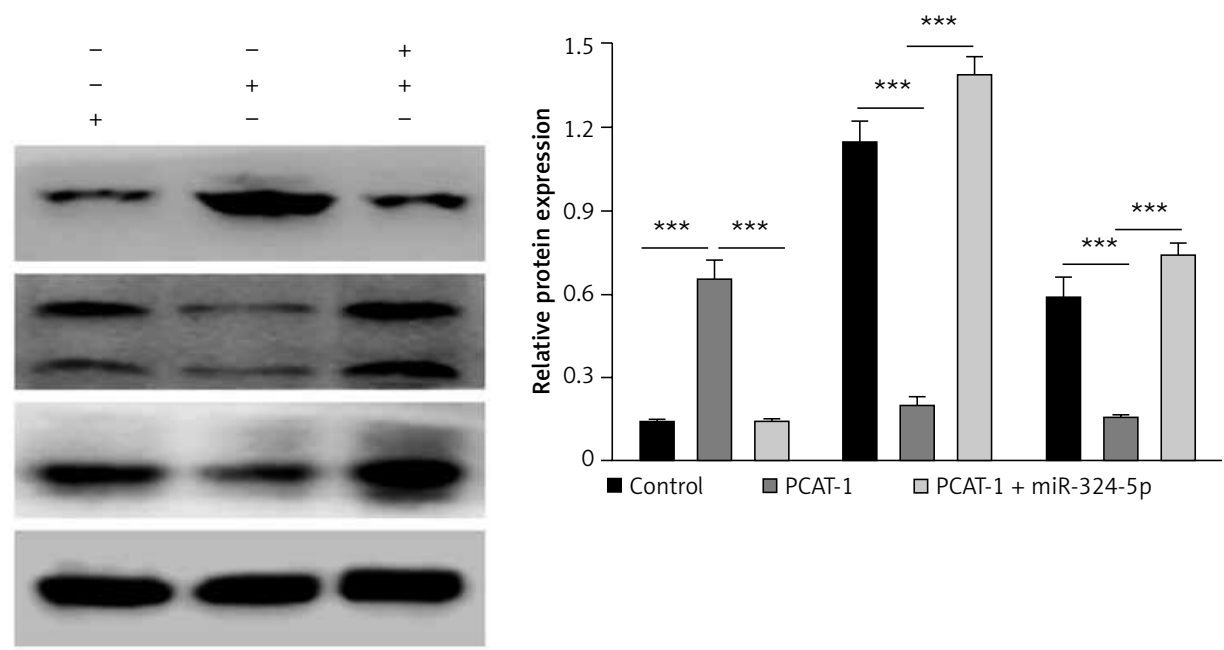

Figure 4. IncRNA PCAT-1 promotes proliferation and inhibits apoptosis of lung cancer cells by downregulating miR324-5p. A - The protein level of RAP1A in A549 cells after transfection with PCAT-1 expressing plasmid (PCAT-1), PCAT-1 expressing plasmid plus miR-324-5p mimics (PCAT-1+miR-324-5p) or the corresponding control, as determined using western blotting. B - Growth curves of A549 cells after transfection with PCAT-1 expressing plasmid (PCAT-1), PCAT-1 expressing plasmid plus miR-324-5p mimics (PCAT-1 + miR-324-5p) or the corresponding control. Measurements of the cell growth rate were tested using a CCK-8 kit. The experiments were performed three times. C - Cell apoptosis analysis of A549 cells after transfection with PCAT-1 expressing plasmid (PCAT-1), PCAT-1 expressing plasmid plus miR-324-5p mimics (PCAT-1 + miR-324-5p) or the corresponding control (left). The proportion of cell apoptosis was analyzed and presented as a histogram (right). The experiments were repeated three times. D - The protein level of Bcl-2, Cleaved caspase-3 and Cleaved PARP in A549 cells after transfection with PCAT-1 expressing plasmid (PCAT-1), PCAT-1 expressing plasmid plus miR-324-5p mimics (PCAT-1 + miR-324-5p) or the corresponding control, as determined using western blotting (left). Relative quantitative results were determined by Image $J$ and shown as a histogram (right)

The data are expressed as mean \pm SEM, ${ }^{* * *} p<0.001$. 
an endogenous sponge for miR-324-5p in cervical cancer cells [14]. Although miR-324-5p overexpression has been revealed in lung cancer cells, whether it can be regulated by IncRNA remains to be illustrated [18]. Our data in the present study showed that PCAT-1 could probably interact with miR-324-5 $p$ and negatively regulate the expression of miR-324-5p. As a class of small noncoding RNAs, miRNAs usually exert their regulatory activity by negatively regulating the target genes. Thus, we speculated that the process of PCAT-1/ miR-324-5p in controlling cell proliferation and cell apoptosis may be involved in the regulation of miR-324-5p's target gene. We next found that miR-324-5p could regulate the expression of RAP1A in lung cancer by targeting its $3^{\prime}$-UTR.

RAP1A is one of the two isoforms of RAP1, which has been implicated in regulating microtubule dynamics and activating the MAPK/ERK pathway, further playing a vital function in cancer progression $[20,21]$. Our results revealed that miR-324-5p can influence cell proliferation and apoptosis though suppressing the expression of RAP1A in lung cancer by targeting its 3'-UTR. Except for miRNA-324-5p, it has been reported that the expression of RAP1A can be regulated by some miRNAs in other disease, which mainly include miR-196a, miR-203 and miR-433 [34-36]. Among these three miRNAs, miR-196a has been found to be upregulated both in non-small cell lung cancer tissue samples and cell lines, and can promote cell proliferation and invasion through targeting HOXA5, suggesting that it might not target RAP1A in lung cancer [37]. Additionally, Chi et al. demonstrated that miR-203 was decreased and could inhibit cell proliferation and migration of non-smallcell lung cancer [38]. The roles of miR-433 in lung cancer still remain unclear. As mentioned above, it was revealed that RAP1A could function as a target gene of miRNAs and play an important role in the development of cancers. Due to the network form of noncoding RNA regulation, further studies are necessary to elucidate whether miR-324-5p, miR-203 and miR-433 could simultaneously target RAP1A in lung cancer. Recently, it was reported that for non-small cell lung cancer patients with EGFR mutation, who have brain metastases, the appropriate treatment methods or timing to use epidermal growth factor receptor (EGFR) tyrosine kinase inhibitors (TKIS) and brain radiation treatment (RT) is urgently needed [39]. Thus, elucidating the biological effects and molecular mechanism of vital IncRNAs in lung cancer may help to better understand the pathological process and develop optimal precision treatment strategies.

In conclusion, our findings revealed that overexpression of IncRNA PCAT-1 can promote cell proliferation and inhibit apoptosis of A549 cells.
Additionally, PCAT-1 could bind to miR-324-5p and reduce its expression. Furthermore, PCAT can competitively sponge miR-324-5p and PCAT-1/miR324-5p played a vital function in controlling the expression of RAP1A in lung cancer by targeting its $3^{\prime}$-UTR. Based on the dual function of pro-proliferation and anti-apoptosis, the signaling axis of IncRNA PCAT-1/miR-324-5p/RAP1A may hold promise as a therapeutic target in lung cancer.

\section{Acknowledgments}

This work was supported by the Special Scientific Research Fund of The First Affiliated Hospital, Chengdu Medicine University. Project name: IL-27 regulating STAT in the molecular mechanism of lung cancer (CYFY2017GLPHX03/) and The role of miRNA-21 in TGF- $\beta 1 /$ Smad7 signaling pathway in pulmonary fibrosis (CYFY2017GLPHX01).

\section{Conflict of interest}

The authors declare no conflict of interest.

\section{References}

1. Siegel RL, Miller KD, Jemal A. Cancer Statistics, 2017. CA Cancer J Clin 2017; 67: 7-30.

2. Cancer Genome Atlas Research N. Comprehensive molecular profiling of lung adenocarcinoma. Nature 2014; 511: 543-50.

3. Schmitt AM, Chang HY. Gene regulation: long RNAs wire up cancer growth. Nature 2013; 500: 536-7.

4. Prensner JR, Chen W, Han S, et al. The long non-coding RNA PCAT-1 promotes prostate cancer cell proliferation through cMyc. Neoplasia 2014; 16: 900-8.

5. Ge X, Chen Y, Liao X, et al. Overexpression of long noncoding RNA PCAT-1 is a novel biomarker of poor prognosis in patients with colorectal cancer. Med Oncol 2013; 30: 588.

6. Bi M, Yu H, Huang B, Tang C. Long non-coding RNA PCAT-1 over-expression promotes proliferation and metastasis in gastric cancer cells through regulating CDKN1A. Gene 2017; 626: 337-43.

7. Zhang F, Wan M, XU Y, et al. Long noncoding RNA PCAT1 regulates extrahepatic cholangiocarcinoma progression via the Wnt/beta-catenin-signaling pathway. Biomed Pharmacother 2017; 94: 55-62.

8. Yan TH, Yang H, Jiang JH, et al. Prognostic significance of long non-coding RNA PCAT-1 expression in human hepatocellular carcinoma. Int J Clin Exp Pathol 2015; 8: 4126-31.

9. Zhao B, Hou X, Zhan H. Long non-coding RNA PCAT-1 over-expression promotes proliferation and metastasis in non-small cell lung cancer cells. Int J Clin Exp Med 2015; 8: 18482-7.

10. Rupaimoole R, Slack FJ. MicroRNA therapeutics: towards a new era for the management of cancer and other diseases. Nat Rev Drug Discov 2017; 16: 203-22.

11. Liu HN, Qie P, Yang G, Song YB. miR-181b inhibits chemoresistance in cisplatin-resistant $\mathrm{H} 446$ small cell lung cancer cells by targeting Bcl-2. Arch Med Sci 2018; 14: 745-51.

12. Chen $\mathrm{Y}$, Wang SX, Mu R, et al. Dysregulation of the miR324-5p-CUEDC2 axis leads to macrophage dysfunction 
and is associated with colon cancer. Cell Rep 2014; 7 : 1982-93.

13. Gu C, Zhang M, Sun W, Dong C. Up-regulation of miR324-5p inhibits proliferation and invasion of colorectal cancer cells by targeting ELAVL1. Oncol Res 2019; 27: 515-24.

14. Jiang $H$, Huang $G$, Zhao $N$, et al. Long non-coding RNA TPT1-AS1 promotes cell growth and metastasis in cervical cancer via acting AS a sponge for miR-324-5p. J Exp Clin Cancer Res 2018; 37: 169.

15. Lin H, Zhou AJ, Zhang JY, Liu SF, Gu JX. MiR-324-5p reduces viability and induces apoptosis in gastric cancer cells through modulating TSPAN8. J Pharm Pharmacol 2018; 70: 1513-20.

16. Song L, Liu D, Zhao Y, et al. Sinomenine inhibits breast cancer cell invasion and migration by suppressing NF-kappaB activation mediated by IL-4/miR-324-5p/ CUEDC2 axis. Biochem Biophys Res Commun 2015; 464: 705-10.

17. Zhang L, Liu D, Tang B, et al. MicroRNA-324-5p suppress es the migration and invasion of MM cells by inhibiting the SCF(beta-TrCP) E3 ligase. Oncol Lett 2018; 16: 5331-8.

18. Lin MH, Chen YZ, Lee MY, et al. Comprehensive identification of microRNA arm selection preference in lung cancer: miR-324-5p and -3p serve oncogenic functions in lung cancer. Oncol Lett 2018; 15: 9818-26.

19. Militello R, Colombo MI. Small GTPases as regulators of cell division. Commun Integr Biol 2013; 6: e25460.

20. Fujita H, Fukuhara S, Sakurai A, et al. Local activation of Rap1 contributes to directional vascular endothelial cell migration accompanied by extension of microtubules on which RAPL, a Rap1-associating molecule, localizes. J Biol Chem 2005; 280: 5022-31.

21. Pizon V, Baldacci G. Rap1A protein interferes with various MAP kinase activating pathways in skeletal myogenic cells. Oncogene 2000; 19: 6074-81.

22. Bailey CL, Kelly P, Casey PJ. Activation of Rap1 promotes prostate cancer metastasis. Cancer Res 2009; 69: 4962-8.

23. Sayyah J, Bartakova A, Nogal N, Quilliam LA, Stupack DG, Brown JH. The Ras-related protein, Rap1A, mediates thrombin-stimulated, integrin-dependent glioblastoma cell proliferation and tumor growth. J Biol Chem 2014; 289: 17689-98.

24. Du L, Subauste MC, DeSevo C, et al. miR-337-3p and its targets STAT3 and RAP1A modulate taxane sensitivity in non-small cell lung cancers. PLoS One 2012; 7: e39167.

25. Ma G, Tang M, Wu Y, Xu X, Pan F, Xu R. LncRNAs and miRNAs: potential biomarkers and therapeutic targets for prostate cancer. Am J Transl Res 2016; 8: 5141-50.

26. Aguiar PN Jr, De Mello RA, Hall P, Tadokoro H, Lima Lopes G. PD-L1 expression as a predictive biomarker in advanced non-small-cell lung cancer: updated survival data. Immunotherapy 2017; 9: 499-506.

27. Jiang $\mathrm{N}$, Meng $\mathrm{X}, \mathrm{Mi} \mathrm{H}$, et al. Circulating IncRNA XLOC_009167 serves as a diagnostic biomarker to predict lung cancer. Clin Chim Acta 2018; 486: 26-33.

28. Leng Q, Tsou JH, Zhan M, Jiang F. Fucosylation genes as circulating biomarkers for lung cancer. J Cancer Res Clin Oncol 2018; 144: 2109-15.

29. Li S, Yang R, Sun X, et al. Identification of SPP1 as a promising biomarker to predict clinical outcome of lung adenocarcinoma individuals. Gene 2018; 679: 398-404.

30. Rafiee A, Riazi-Rad F, Havaskary M, Nuri F. Long noncoding RNAs: regulation, function and cancer. Biotechnol Genet Eng Rev 2018; 34: 153-80.
31. Huang Z, Lei W, Tan J, Hu HB. Long noncoding RNA LINC00961 inhibits cell proliferation and induces cell apoptosis in human non-small cell lung cancer. J Cell Biochem 2018; 119: 9072-80.

32. Cheng D, Bao C, Zhang X, Lin X, Huang H, Zhao L. LncRNA PRNCR1 interacts with HEY2 to abolish miR-448-mediated growth inhibition in non-small cell lung cancer. Biomed Pharmacother 2018; 107: 1540-7.

33. Tang LX, Chen GH, Li H, He P, Zhang Y, Xu XW. Long non-coding RNA OGFRP1 regulates LYPD3 expression by sponging miR-124-3p and promotes non-small cell lung cancer progression. Biochem Biophys Res Commun 2018; 505: 578-85.

34. Wang K, Li J, Guo H, et al. MiR-196a binding-site SNP regulates RAP1A expression contributing to esophageal squamous cell carcinoma risk and metastasis. Carcinogenesis 2012; 33: 2147-54.

35. Xiang J, Bian C, Wang H, Huang S, Wu D. MiR-203 downregulates Rap1A and suppresses cell proliferation, adhesion and invasion in prostate cancer. J Exp Clin Cancer Res 2015; 34: 8.

36. Zhang T, Jiang $\mathrm{K}$, Zhu X, et al. miR-433 inhibits breast cancer cell growth via the MAPK signaling pathway by targeting Rap1a. Int J Biol Sci 2018; 14: 622-32.

37. Liu XH, Lu KH, Wang KM, et al. MicroRNA-196a promotes non-small cell lung cancer cell proliferation and invasion through targeting HOXA5. BMC Cancer 2012; 12: 348.

38. Chi Y, Jin Q, Liu X, et al. miR-203 inhibits cell proliferation, invasion, and migration of non-small-cell lung cancer by downregulating RGS17. Cancer Sci 2017; 108: 2366-72.

39. Wang W, Song Z, Zhang Y. Efficacy of brain radiotherapy plus EGFR-TKI for EGFR-mutated non-small cell lung cancer patients who develop brain metastasis. Arch Med Sci 2018; 14: 1298-307. 